\title{
ANALYSIS OF MASS TRANSFER IN HOLLOW-FIBER MEMBRANE SEPARATOR VIA NONLINEAR EIGENFUNCTION EXPANSIONS
}

\author{
Péricles C. Pontes, ${ }^{1,2}$ Anderson P. Almeida, ${ }^{1}$ \\ Renato M. Cotta, ${ }^{1,3,4, *}$ E Carolina P. Naveira-Cotta ${ }^{1,4}$ \\ ${ }^{1}$ Laboratory of Nano- and Microfluidics and Microsystems, LabMEMS, \\ Mechanical Engineering Department and Nanotechnology Engineering Dept., \\ POLI \& COPPE, Universidade Federal do Rio de Janeiro, Cidade Universitária, \\ Cx. Postal 68503, Rio de Janeiro, RJ, CEP 21945-970, Brazil \\ ${ }^{2}$ Araguaia Institute of Engineering, Federal University of South and Southeast \\ of Pará-UNIFESSPA, Santana do Araguaia, PA, Brazil \\ ${ }^{3}$ Interdisciplinary Nucleus for Social Development-NIDES/CT, UFRJ, Brazil \\ ${ }^{4}$ Mechanical Engineering Department, University College London, UCL, United \\ Kingdom
}

*Address all correspondence to: Renato M. Cotta, Laboratory of Nano- and Microfluidics and Microsystems, LabMEMS, Mechanical Engineering Department and Nanotechnology Engineering Dept., POLI \& COPPE, Universidade Federal do Rio de Janeiro, Cidade Universitária, Cx. Postal 68503, Rio de Janeiro, RJ, CEP 21945-970, Brazil, E-mail: cotta@mecanica.coppe.ufrj.br

The Generalized Integral Transform Technique (GITT) is a well-established hybrid numericalanalytical method applicable to the solution of linear or nonlinear convection-diffusion problems, which presents relatively low computational cost and automatic error control. Here, this hybrid method is employed in the analysis of mass transfer in hollow-fiber mass separators. The adopted model considers fully developed laminar flow of a Newtonian fluid with diffusion and reaction transport effects of the solute through the membrane pores. The diffusive-reactive process at the membrane is represented through a nonlinear boundary condition. A hybrid numerical-analytical solution is obtained, based on retaining the original nonlinear boundary condition coefficients in the eigenvalue problem proposition. The developed nonlinear eigenfunction expansion is then thoroughly analyzed in terms of convergence behavior. The novel approach is also critically compared against previously reported numerical results for typical parametric values and with an alternative convergence enhancement approach based on the proposition of an implicit nonlinear filter, that makes the boundary condition homogeneous and allows for an integral transform solution through the proposition of a linear eigenvalue problem.

KEY WORDS: convection-diffusion-reaction, separation, membrane separator, nonlinear eigenvalue problem, nonlinear filter, integral transforms 


\begin{tabular}{|c|c|c|c|}
\hline \multicolumn{4}{|c|}{ NOMENCLATURE } \\
\hline$C$ & dimensionless concentration & $\mathrm{Sh}_{w}$ & wall Sherwood number \\
\hline $\bar{C}$ & integral transformed dimensionless & $U$ & dimensionless velocity profile \\
\hline & concentration & $u$ & dimensional velocity profile, $\mathrm{m} \mathrm{s}^{-1}$ \\
\hline$C^{*}$ & dimensional concentration, $\mathrm{mol} \mathrm{m}^{-3}$ & $u_{m}$ & average fluid velocity, $\mathrm{m} \mathrm{s}^{-1}$ \\
\hline$C_{T}$ & $\begin{array}{l}\text { total carrier concentration in all forms } \\
(B \text { and } A B)\end{array}$ & $\begin{array}{l}z \\
z^{*}\end{array}$ & $\begin{array}{l}\text { dimensionless axial coordinate } \\
\text { dimensional axial coordinate, } \mathrm{m}\end{array}$ \\
\hline$D$ & $\begin{array}{l}\text { diffusivity of solute in the fluid phase, } \\
\mathrm{m}^{2} \mathrm{~s}^{-1}\end{array}$ & & Greek symbols \\
\hline$H$ & $\begin{array}{l}\text { equilibrium distribution coefficient of } \\
\text { solute concentration in the membrane }\end{array}$ & $\gamma$ & $\begin{array}{l}\text { dimensionless slope for a variable } \\
\text { distribution coefficient }\end{array}$ \\
\hline & to that in the fluid & $\alpha$ & maximum facilitation factor \\
\hline$h^{*}$ & $\begin{array}{l}\text { dimensional slope of the distribution } \\
\text { coefficient, } \mathrm{m}^{3} \mathrm{~mol}^{-1}\end{array}$ & $\begin{array}{l}\beta \\
\Psi\end{array}$ & $\begin{array}{l}\text { dimensionless equilibrium constant } \\
\text { eigenfunction }\end{array}$ \\
\hline$h_{o}$ & distribution coefficient for infinite & $\tilde{\Psi}$ & normalized eigenfunction \\
\hline$K_{e q}$ & $\begin{array}{l}\text { solute dilution } \\
\text { equilibrium constant, ratio of the }\end{array}$ & $\mu$ & eigenvalues \\
\hline & forward to the backward rate constants & & Subscripts and superscripts \\
\hline$k_{m}$ & $\begin{array}{l}\text { membrane permeability coefficient, } \\
\mathrm{m} \mathrm{s}^{-1}\end{array}$ & $\begin{array}{l}e \\
f\end{array}$ & $\begin{array}{l}\text { entrance position } \\
\text { filter solution }\end{array}$ \\
\hline $\begin{array}{l}R \\
R_{o}\end{array}$ & $\begin{array}{l}\text { inner radius of hollow fiber, } \mathrm{m} \\
\text { outer radius of hollow fiber, } \mathrm{m}\end{array}$ & $i, j$ & $\begin{array}{l}\text { order of eigenvalues and } \\
\text { eigenfunctions }\end{array}$ \\
\hline$r$ & dimensionless radial coordinate & $h$ & homogeneous solution \\
\hline$r^{*}$ & dimensional radial coordinate, $\mathrm{m}$ & $A$ & referring to the species A \\
\hline$s$ & hollow fiber shape factor & $B$ & referring to the species B \\
\hline
\end{tabular}

\section{INTRODUCTION}

Mass separators of hollow fiber membranes have application in several areas, such as in dialysis, metal or non-dispersive solvent extraction, gas separation, artificial oxygenation, and removal of pollutants from industrial waste streams (Urtiaga et al., 1992). This device is formed by a large number of modules containing several synthetic tubular membranes that can promote the separation of two or more phases, restricting the transport of many chemical species and selectively transporting other species (Porter, 1990).

There is great interest in the study of supported liquid membranes because they can promote the selective separation of a solute between two aqueous solutions. The procedure consists of immobilizing an organic liquid (solvent) in the micropores within the porous structure of the membrane, to promote the transfer of the solute by the membrane through diffusion, accompanied or not by chemical reaction (Cardoso et al., 2009; Kim and Stroeve, 1988, 1989, 1990; Urtiaga et al., 1992).

Supported liquid membranes can be applied in various processes such as separation of metals (Cardoso et al., 2009; Lakshmi et al., 2004; Swain et al., 2004), wastewater purification (Hosseini et al., 2016), and pervaporation (Rdzaneck et al., 2018). This technology offers advantages as facilitated transport mechanism, high selectivity, easy scale-up, low energy demand, and low operational cost, and it is an alternative to the traditional separation and purification processes 
(Kocherginsky et al., 2007). However, the use of this technology on an industrial scale is still reduced due to instability problems of the organic phase impregnated in the porous structure of the membrane, whose losses can contaminate the stripping solution and reduce the separation efficiency (Kocherginsky et al., 2007).

The mass transfer rate is limited by the transport of the solute through the membrane and it is characterized by the wall Sherwood number $\left(\mathrm{Sh}_{w}\right)$, which is defined as the ratio of the mass transfer resistance in the fluid to that in the membrane. If $\mathrm{Sh}_{w} \rightarrow \infty$ then the mass transfer resistance in the membrane can be neglected and the solute concentration in the membrane wall can be considered constant, and the problem formulation becomes similar to the classical Graetz problem in heat transfer analysis. For $\mathrm{Sh}_{w}=0$, the resistance in the membrane is dominant and the problem has a prescribed flux condition at the fluid-membrane interface. High values of $\mathrm{Sh}_{w}$ ensure improved mass transfer rates through the membrane and a high selectivity for the separation process. For a fluid with several components, it is necessary to choose a membrane that allows a higher Sherwood number for the component to be separated in comparison to the others. A relatively high Sherwood number can be obtained by using liquid membranes containing a suitable liquid to ensure a high distribution coefficient for the solute (Urtiaga et al., 1992).

The mass transfer process can also be improved through the use of carrier species which selectively and reversibly react with the solute by promoting its transport through the porous liquid membrane. These carrier species should be retained in the membrane due to limitations of solubility. This transport favored by a carrier species is known as carrier-facilitated transport (Kim and Stroeve, 1988).

Mathematical models that govern the mass transfer process in hollow fiber membranes involving chemical reaction are generally nonlinear due to reaction kinetics and, therefore, require the application of numerical or hybrid analytical-numerical methods to construct the solution. Hybrid methods are in general advantageous over purely numerical methods since they explore known analytical ideas within the computational procedure, in an attempt to achieve results of higher accuracy and lower computational cost, and are naturally preferable in time-consuming computational tasks such as optimization and inverse problem analysis.

The Generalized Integral Transform Technique (GITT) is a well-established tool in the hybrid numerical-analytical solution of various classes of linear and nonlinear diffusion and convection-diffusion problems (Cotta, 1990, 1993, 1994, 1998; Cotta and Mikhailov, 1997, 2006; Cotta et al., 2016a). This method can be viewed as an extension of the Classical Integral Transform Technique (Kakaç et al., 2018; Mikhailov and Ozisik, 1984; Özisik, 1993) and consists of constructing the solution to the desired potentials as an expansion of orthogonal eigenfunctions, obtained from the proposition of a suitable eigenvalue problem, that, as much as possible, should incorporate information on the original problem for improved convergence.

In most of the previous implementations of this approach, linear eigenvalue problems have been proposed in providing the basis for the eigenfunction expansions. Typically, the original nonlinear problem formulation is first rewritten by retaining characteristic linear coefficients in the transient, diffusive, and dissipation operators of the partial differential equations, while transporting the remaining nonlinear terms to a rewritten nonlinear equation source term. Again, the same formulation interpretation is adopted in case those nonlinear boundary conditions are present. Then, such characteristic equation and boundary condition linear coefficients naturally lead to the eigenvalue problem choice to be employed in constructing the expansions. Recently, a variant in the GITT approach has been advanced, based on retaining the original nonlinear operator coefficients in the eigenvalue problem proposition (Cotta et al., 2016b). This methodology has been demonstrated in diffusion problems with nonlinear boundary conditions, which

Volume 30, Issues 2-3, 2018 
(Cotta et al., 2015, 2016b; Pontes et al., 2017) clearly illustrate the relative gains in convergence enhancement in comparison to other alternative convergence acceleration schemes, such as filtering and integral balances.

The present contribution is focused on the analysis of the convective-diffusive mass transfer related to the transport of a solute through polymeric hollow fiber membranes, undergoing diffusive or diffusive-reactive separation, as modeled through a nonlinear boundary condition. The novel GITT variant with nonlinear eigenfunction expansion is then employed, and critically compared to the more traditional approach of employing a linear eigenvalue problem basis but adopting a nonlinear filter scheme for convergence enhancement. The aim is to analyze the computational performance achieved by both approaches, before selecting the most adequate solution path for a broader physical analysis. Then, the influence of the governing parameters on the mass separation process in tubular membranes is investigated. Comparisons are also performed against results from previously reported numerical implementations, in order to verify the algorithms and demonstrate the potential of this technique in dealing with such class of nonlinear problems.

\section{PROBLEM FORMULATION}

The mass separator will be studied assuming that each hollow fiber membrane within the device exhibits a similar behavior, and thus assuming that modeling the mass transfer process for one single membrane is then sufficient to represent the overall physical performance of the separator. The membranes are suitably supported on the porous walls of the hollow fiber whereby the solute is transported by diffusion or diffusion-reaction, according to the characteristics of the material used in the membrane composition and its affinity with the solute. A schematic diagram of the hollow fiber membrane is shown in Fig. 1.

The partial differential equations that govern the mass transfer process in the mass separator were presented in the literature (Kim and Stroeve, 1988, 1989; Urtiaga et al., 1992), based on the mass conservation principle, assuming steady-state fully developed laminar flow of a Newtonian fluid containing the solute to be separated through the hollow fiber membranes, by diffusion or diffusion-reaction effects. The fluid enters the separator with known uniform concentration $C_{e}^{*}$ and the separation process starts at $z=0$, where the fluid comes into contact with the supported

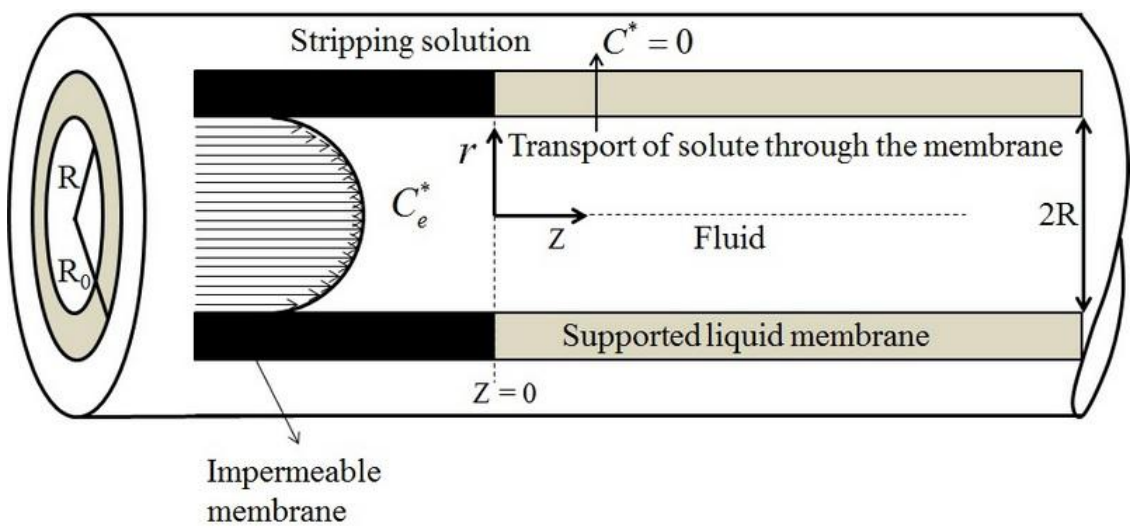

FIG. 1: Schematic representation of the hollow fiber geometry and coordinates system, with the supported liquid membrane 
liquid membrane. The solute permeates through the liquid membrane by diffusion or diffusionreaction process, and on the other side of the fiber reacts instantaneously with the stripping solution, so that its concentration is equal to zero. The axial diffusion effect is neglected compared to axial convection and radial diffusion. The dimensionless mathematical model is thus given by

$$
\begin{gathered}
U(r) \frac{\partial C(r, z)}{\partial z}=\frac{1}{r} \frac{\partial}{\partial r}\left(r \frac{\partial C(r, z)}{\partial r}\right), 0<r<1, z>0 \\
C(r, 0)=1,0<r<1 \\
\left.\frac{\partial C(r, z)}{\partial r}\right|_{r=0}=0, z>0 \\
\left.\frac{\partial C(r, z)}{\partial r}\right|_{r=1}+f(C) C(1, z)=0, z>0
\end{gathered}
$$

where $r=r^{*} / R$ is the dimensionless radial coordinate, $z=z^{*} D / u_{m} R^{2}$ is the dimensionless axial coordinate, $C=C^{*} / C_{e}^{*}$ is the dimensionless concentration of the solute, and $U(r)=$ $2\left(1-r^{2}\right)$ is the dimensionless velocity profile of the fluid.

Equation (1c) represents the symmetry condition at the channel center line, while Eq. (1d) is a nonlinear boundary condition that imposes the continuity of solute flux across the membranefluid interface, which makes it unlikely to obtain a fully analytical solution for this problem. In this case, a reliable numerical or hybrid numerical-analytical method should be employed to obtain an accurate solution.

The term $f(C)$ in the boundary condition Eq. (1d) is defined according to the assumptions adopted for the transport of the solute through the membrane. For instance, Urtiaga et al. (1992) assumed a linear dependency of the equilibrium distribution coefficient on the solute concentration. The equilibrium distribution coefficient is defined as the equilibrium distribution ratio of the solute concentration in the liquid membrane to the concentration in the fluid side. In this case, the solute is separated only by diffusion through the membrane, and the term $f(C)$ and the dimensionless groups are defined by Eqs. (1e)-(1g), respectively:

$$
\begin{gathered}
f(C)=\operatorname{Sh}_{w}(1+\gamma C(1, z)) \\
\gamma=\frac{C_{e}^{*} h^{*}}{h_{o}} \\
\operatorname{Sh}_{w}=\frac{k_{w} s R h_{o}}{D}
\end{gathered}
$$

where $h_{o}$ is the value of the distribution coefficient for infinite dilute solutions and $s=\left(R_{o}-R\right) /$ $\left(R \ln \left[R_{o} / R\right]\right)$ is the shape factor based on the inside radius (Noble, 1983).

The mathematical model proposed by Urtiaga et al. (1992) can be applied in the analysis of the separation process of toxic phenolic solutes from water through liquid membranes, for which some solvents have a distribution coefficient depending on the concentration of phenol.

Kim and Stroeve (1988) considered that the solute may be transported through the membrane by carrier-facilitated transport, promoted by a complexation reaction with carrier species contained in the membrane, which provide improved mass transfer rates and high selectivity. The solute-carrier complex $A B$ is obtained from an elementary and reversible equilibrium reaction between the solute (species $A$ ) and carrier (species $B$ ) inside the membrane, with the

Volume 30, Issues 2-3, 2018 
kinetics represented in Eq. (1h). The term $f(C)$ and the dimensionless groups can be defined by Eqs. (1i)-(1m), respectively.

$$
\begin{gathered}
A+B \rightleftarrows A B \\
f(C)=\mathrm{Sh}_{w}\left(1+\frac{\alpha}{1+\beta C(1, z)}\right) \\
z=\frac{z^{*} D_{A}}{u_{m} R^{2}} \\
\mathrm{Sh}_{w}=\frac{k_{w} s R}{D_{A}} \\
\alpha=\frac{D_{B}^{\prime} C_{T} K_{e q}}{D_{A}^{\prime}} \\
\beta=K_{e q} H C_{e}^{*}
\end{gathered}
$$

The mathematical model proposed by Kim and Stroeve (1988) can be applied in the removal process of $\mathrm{CO}_{2}$ from synthesis gas, using a reactive carbonate solution immobilized in microporous polymeric membranes.

Kim and Stroeve (1989) also considered that the solute can be transported through the membrane by carrier-facilitated ion-pair transport, promoted by an ion-pair formation and complexation reaction. First, the cation $A$ (solute to be separated) reacts with the anion $B$ leading to the species $A B$, with kinetics defined by Eq. (1n). Then, the complex $A B P$ is obtained from the reaction between the species $A B$ and the carrier $P$, with kinetics defined by Eq. (1o). Both reactions are considered elementary and reversible. The term $f(C)$ and the dimensionless groups are defined by Eqs. (1p)-(1t), respectively,

$$
\begin{gathered}
A+B \rightleftarrows A B \\
A B+P \rightleftarrows A B P \\
f(C)=\mathrm{Sh}_{w}\left(1+\frac{\alpha}{1+\beta C^{2}(1, z)}\right) C(1, z) \\
z=\frac{z^{*} D_{A}}{v R^{2}} \\
\mathrm{Sh}_{w}=\frac{k_{w A B} s k C_{e}^{*}}{D_{A}} \\
\alpha=\frac{D_{C}^{\prime} C_{T} K_{e q}}{D_{A B}^{\prime}} \\
\beta=K_{e q} h\left(C_{e}^{*}\right)^{2}
\end{gathered}
$$

The mathematical model proposed by Kim and Stroeve (1989) can be applied in the transport of mono- and divalent cations through chloroform-saturated membranes containing crown ether carriers. 


\section{SOLUTION METHODOLOGY}

As an alternative to conventional numerical methods, the hybrid numerical-analytical approach known as GITT (Generalized Integral Transform Technique) (Cotta, 1990, 1993, 1994, 1998; Cotta and Mikhailov, 1997, 2006; Cotta et al., 2016a) will be used to construct the solution of the given problem, as an expansion of orthogonal eigenfunctions defined from an appropriate eigenvalue problem. Following the solution of the eigenvalue problem, the dependence of the radial variable $r$ is then removed through integral transformation of the partial differential equation, leading to a system of coupled ordinary differential equations which must be solved numerically for the transformed concentrations.

Two variants of the eigenfunction expansion will be here analyzed. First, as a major goal of the present work, a nonlinear eigenvalue problem will be adopted, which incorporates the nonlinear diffusion-reaction effects at the interface fluid-membrane. In this first solution variant, the circular geometry and velocity profile are not accounted for by the eigenvalue problem choice, so as to provide simpler eigenfunctions. It incorporates the boundary condition nonlinearity, generating nonlinear ordinary differential equations for the determination of eigenvalues, to be solved simultaneously with the transformed system. In the second solution variant, both the circular geometry and the velocity profile are incorporated into the eigenvalue problem formulation, which yields Laguerre polynomials as the basis for the expansion, but without incorporating the boundary nonlinearity, which must then be a priori homogenized by the application of an implicit nonlinear filter, within a constrained range of the governing parameters.

\subsection{Nonlinear Eigenvalue Problem Approach}

Here, a recently introduced integral transforms approach (Cotta et al., 2016b), based on the adoption of nonlinear eigenvalue problems, will be further investigated. There is some computational advantage, as will be seen further ahead, in adopting a simpler eigenvalue problem formulation that does not account for the circular geometry and velocity profile information, but instead directly incorporates the nonlinear boundary condition information. Following this alternative, the proposed nonlinear eigenvalue problem is given by

$$
\begin{gathered}
\frac{\partial^{2} \Psi_{i}}{\partial r^{2}}+\mu_{i}^{2}(z) \Psi_{i}(r ; z)=0 \\
\left.\frac{\partial \Psi_{i}}{\partial r}\right|_{r=0}=0 \\
\left.\frac{\partial \Psi_{i}}{\partial r}\right|_{r=1}+f(C) \Psi_{i}(1 ; z)=0
\end{gathered}
$$

Making use of the orthogonality property of the eigenfunctions, the following integral transform pair can be defined:

$$
\begin{gathered}
\bar{C}_{i}(z)=\int_{0}^{1} \Psi_{i}(r ; z) C(r, z) d r \text { transform } \\
C(r, z)=\sum_{i=1}^{\infty} \frac{\Psi_{i}(r ; z)}{N_{i}(z)} \bar{C}_{i}(z) \text { inverse }
\end{gathered}
$$

Volume 30, Issues 2-3, 2018 
where the kernels $\Psi_{i}(r ; z)$ are given by the solution of the eigenvalue problem, Eqs. (2a) and (2b). This eigenvalue problem has a known analytical solution given by

$$
\Psi_{i}(r ; z)=\cos \left(\mu_{i}(z) r\right)
$$

with $N_{i}(z)$, the normalization integral, given by

$$
N_{i}(z)=\int_{0}^{1} \Psi_{i}^{2}(r ; z) d r=\frac{1}{4}\left(2+\frac{\sin \left(2 \mu_{i}(z)\right)}{\mu_{i}(z)}\right)
$$

The proposed eigenvalue problem is typical of diffusion problems in Cartesian coordinates, but it was chosen to allow for the analytical solution of the integrals obtained along the integral transformation procedure, thus avoiding costly numerical integrations.

The integral transformation of Eq. (1a) is accomplished by applying the operator $\int_{0}^{1} \Psi_{i}(r ; z)() d$.$r and making use of the boundary conditions given by Eqs. (1c), (1d), (2b), and$ (2c), yielding the transformed system of ordinary differential equations below:

$$
\sum_{j=1}^{\infty} A_{i, j}(z) \frac{d \bar{C}_{j}(z)}{d z}=\sum_{j=1}^{\infty}\left(E_{i, j}(z)-B_{i, j}(z)\right) \bar{C}_{j}(z)-\mu_{i}^{2}(z) \bar{C}_{i}(z), \quad i=1,2,3, \ldots
$$

where

$$
\begin{gathered}
A_{i, j}(z)=\frac{1}{N_{j}(z)} \int_{0}^{1} U(r) \Psi_{i}(r ; z) \Psi_{j}(r ; z) d r \\
B_{i, j}(z)=\int_{0}^{1} U(r) \Psi_{i}(r ; z) \frac{\partial}{\partial z}\left(\frac{\Psi_{j}(r ; z)}{N_{j}(z)}\right) d r \\
E_{i, j}(z)=\frac{1}{N_{j}(z)} \int_{0}^{1} \frac{\Psi_{i}(r ; z)}{r} \frac{\partial \Psi_{j}}{\partial r} d r
\end{gathered}
$$

The inlet boundary condition given by Eq. (1b) is transformed through the operator $\int_{0}^{1} \Psi_{i}(r ; 0)() d r$, to provide

$$
\bar{C}_{i}(0)=F_{i}
$$

where

$$
F_{i}=\int_{0}^{1} \Psi_{i}(r ; 0) d r
$$

For the solution of the infinite coupled system of nonlinear ordinary differential equations (ODE) given by Eqs. (5a)-5(f), one usually needs to make use of numerical algorithms, after the truncation of the system to a sufficiently large finite order. For instance, the built-in routine NDSolve of the Mathematica system, Wolfram (2015), may be employed, which is able to provide reliable solutions under automatic absolute and relative errors control. Then, the inversion 
formula, Eq. (3b), can be recalled to yield the concentration field representation at any desired position $r$ and $z$.

Replacing Eq. (4a), obtained for the eigenfunction $\Psi_{i}(r ; z)$, into the nonlinear boundary condition, Eq. (2c), one may reach the transcendental equation for $\mu_{i}(z)$ :

$$
-\mu_{i}(z) \sin \left(\mu_{i}(z)\right)+f(C(1, z)) \cos \left(\mu_{i}(z)\right)=0
$$

Taking the derivative of Eq. (6a), it is obtained an ODE system for $\mu_{i}(z)$, in the form:

$$
\frac{d \mu_{i}(z)}{d z}=\frac{\cos \left(\mu_{i}(z)\right) d f / d z}{\sin \left(\mu_{i}(z)\right)+\mu_{i}(z) \cos \left(\mu_{i}(z)\right)+f(C(1, z)) \sin \left(\mu_{i}(z)\right)}, \quad i=1,2,3, \ldots
$$

The derivative of $f(C(1, z))$ is evaluated for each model as

- Urtiaga et al. (1992):

$$
\frac{d f}{d z}=\operatorname{Sh}_{w} \gamma \frac{\partial C(1, z)}{\partial z}
$$

- Kim and Stroeve (1988):

$$
\frac{d f}{d z}=-\frac{\operatorname{Sh}_{w} \alpha \beta}{(1+\beta C(1, z))^{2}} \frac{\partial C(1, z)}{\partial z}
$$

- Kim and Stroeve (1989):

$$
\frac{d f}{d z}=\operatorname{Sh}_{w}\left[1+\frac{\alpha\left(1-\beta C^{2}(1, z)\right)}{\left(1+\beta C^{2}(1, z)\right)^{2}}\right] \frac{\partial C(1, z)}{\partial z}
$$

where

$$
\begin{aligned}
\frac{\partial C(1, z)}{\partial z} & =\sum_{j=1}^{\infty} \frac{1}{N_{j}^{2}(z)}\left[\left(\frac{d \Psi_{j}(1 ; z)}{d z} N_{j}(z)-\Psi_{j}(1 ; z) \frac{d N_{j}(z)}{d z}\right) \bar{C}_{j}(z)\right. \\
& \left.+\Psi_{j}(1 ; z) N_{j}(z) \frac{d \bar{C}_{j}(z)}{d z}\right]
\end{aligned}
$$

The inlet boundary conditions for the eigenvalues ODE system, Eqs. (6b)-6(f), can be obtained by the evaluation of Eq. (6a) at $z=0$. At $z=0$, there is a prescribed concentration condition, but when computing the eigenvalues for this inlet condition, the inverse formula for the concentration should be employed in Eq. (6a), to be consistent with the substitution performed in deriving Eq. (6f) for its derivative evaluation.

\subsection{Nonlinear Filtering Solution Approach}

Employing a nonlinear (or implicit) filter solution can be a very effective strategy to account for the nonlinear source term and avoid a slower convergence behavior of the eigenfunction expansion (Cotta and Mikhailov, 1997). In order to remove the nonlinearity of the boundary condition [Eq. (1d)], the following nonlinear filter solution has been proposed:

$$
C(r, z)=C_{h}(r, z)+C_{f}(r ; z)
$$

Volume 30, Issues 2-3, 2018 
where $C_{h}(r, z)$ is the homogeneous potential solution and $C_{f}(r ; z)$ is the nonlinear filter solution.

The filter solution is then obtained from the following problem formulation:

$$
\begin{gathered}
\frac{1}{r} \frac{\partial}{\partial r}\left(r \frac{\partial C_{f}(r ; z)}{\partial r}\right)=0 \\
\left.\frac{\partial C_{f}(r ; z)}{\partial r}\right|_{r=0}=0
\end{gathered}
$$

while the nonlinear boundary condition and the filter solution for each model is given as

- Urtiaga et al. (1992):

$$
\begin{gathered}
\left.\frac{\partial C_{f}(r ; z)}{\partial r}\right|_{r=1}+\operatorname{Sh}_{w} C_{f}(1 ; z)=-\operatorname{Sh}_{w} \gamma\left[C_{h}^{2}(1, z)+2 C_{h}(1, z) C_{f}(1 ; z)+C_{f}^{2}(1 ; z)\right] \\
C_{f}(z)=\frac{-\left[1+2 \gamma C_{h}(1, z)\right]+\sqrt{1+4 \gamma C_{h}(1, z)}}{2 \gamma}, \text { if } \gamma \neq 0 \\
C_{f}(z)=0, \text { if } \gamma=0
\end{gathered}
$$

- Kim and Stroeve (1988):

$$
\begin{gathered}
\left.\frac{\partial C_{f}(r ; z)}{\partial r}\right|_{r=1}+\operatorname{Sh}_{w} C_{f}(1 ; z)=-\frac{\operatorname{Sh}_{w} \alpha\left(C_{h}(1, z)+C_{f}(1 ; z)\right)}{1+\beta\left(C_{h}(1, z)+C_{f}(1 ; z)\right)} \\
C_{f}(z)=\frac{-\left(1+\alpha+\beta C_{h}(1, z)\right)+\sqrt{-4 \alpha \beta C_{h}(1, z)+\left[1+\alpha+\beta C_{h}(1, z)\right]^{2}}}{2 \beta} \\
\text { if } \beta \neq 0 \\
C_{f}(z)=-\frac{\alpha C_{h}(1, z)}{(1+\alpha)}, \text { if } \beta=0
\end{gathered}
$$

Since the first boundary condition, Eq. (8b), eliminates the logarithmic radial dependence in the filter, the resulting filter solutions are given by functions of the axial variable only, $C_{f}(z)$. Equations (8d), (8e), (8g), and (8h) establish nonlinear relationships between the filter and the homogeneous concentration at $r=1$, for these particular forms of the boundary source terms here analyzed. It is only possible to obtain a real solution for Eqs. (8d) and $(8 \mathrm{~g})$ if the following conditions are satisfied, respectively:

$$
\begin{gathered}
1+4 \gamma C_{h}(1, z) \geq 0 \\
-4 \alpha \beta C_{h}(1, z)+\left[1+\alpha+\beta C_{h}(1, z)\right]^{2} \geq 0
\end{gathered}
$$

In addition, the mathematical model proposed by Kim and Stroeve (1989) does not provide an explicit relationship for the nonlinear filter, following the same proposal shown above, and is therefore not included in the comparisons that shall be here performed between the two solution variants. 
After finding the filter solution, the problem to determine the homogeneous potential can be analyzed, which is defined by the following equations:

$$
\begin{gathered}
U(r) \frac{\partial C_{h}(r, z)}{\partial z}=\frac{1}{r} \frac{\partial}{\partial r}\left(r \frac{\partial C_{h}(r, z)}{\partial r}\right)+P\left(r, z, C_{h}(1, z)\right) \\
C_{h}(r, 0)=1-C_{f}(0) \\
\left.\frac{\partial C_{h}(r, z)}{\partial r}\right|_{r=0}=0 \\
\left.\frac{\partial C_{h}(r, z)}{\partial r}\right|_{r=1}+\operatorname{Sh}_{w} C_{h}(1, z)=0
\end{gathered}
$$

where

$$
P\left(r, z, C_{h}(1, z)\right)=-U(r) \frac{d C_{f}(z)}{d z}
$$

As it can be observed from Eqs. (9a)-(9e), application of the nonlinear filter results in a linear and homogeneous boundary condition, while a nonlinear source term is created in the partial differential equation, Eq. (9a). The GITT methodology can also be directly applied to this homogeneous problem with a linear boundary condition at $r=1$. For this purpose, the following linear eigenvalue problem was chosen:

$$
\begin{gathered}
\frac{1}{r} \frac{d}{d r}\left(r \frac{d \Psi_{i}}{d r}\right)+\mu_{i}^{2} U(r) \Psi_{i}(r)=0 \\
\left.\frac{d \Psi_{i}}{d r}\right|_{r=0}=0 \\
\left.\frac{d \Psi_{i}}{d r}\right|_{r=1}+\operatorname{Sh}_{w} \Psi_{i}(1)=0
\end{gathered}
$$

The eigenvalue problem above defined has analytical solution in terms of Laguerre polynomials:

$$
\Psi_{i}(r)=e^{-\left(r^{2} \mu_{i} / \sqrt{2}\right)} L_{(1 / 4)\left(-2+\sqrt{2} \mu_{i}\right)}\left(\sqrt{2} r^{2} \mu_{i}\right)
$$

After the eigenvalue problem solution, the transform and inverse formulae for the homogeneous concentration can be defined as

$$
\begin{gathered}
\bar{C}_{h, i}(r)=\int_{0}^{1} r U(r) \tilde{\Psi}_{i}(r) C_{h}(r, z) d r \\
C_{h}(r, z)=\sum_{i=1}^{\infty} \tilde{\Psi}_{i}(r) \bar{C}_{h_{i}}(z)
\end{gathered}
$$

where $\tilde{\Psi}_{i}(r)$ is the normalized eigenfunction defined as

$$
\tilde{\Psi}_{i}(r)=\frac{\Psi_{i}(r)}{\sqrt{N_{i}}}
$$

Volume 30, Issues 2-3, 2018 


$$
N_{i}=\int_{0}^{1} r U(r) \Psi_{i}^{2}(r) d r=\frac{1}{2 \mu_{i}}\left(\frac{\partial \Psi_{i}}{\partial r} \frac{\partial \Psi_{i}}{\partial \mu_{i}}-\Psi_{i} \frac{\partial^{2} \Psi_{i}}{\partial r \partial \mu_{i}}\right)_{r=1}
$$

The integral transformation of Eq. (9a) is accomplished by applying the operator $\int_{0}^{1} r \tilde{\Psi}_{i}(r)() d$.$r and making use of the boundary conditions given by Eqs. (9c), (9d), (10b),$ and $(10 \mathrm{c})$, yielding the transformed system of nonlinear ordinary differential equations:

$$
\frac{d \bar{C}_{h, i}(z)}{d z}+\mu_{i}^{2} \bar{C}_{h, i}(z)=\bar{P}_{i}\left(z, C_{h}(1, z)\right)
$$

where

$$
\bar{P}_{i}\left(z, C_{h}(1, z)\right)=-\int_{0}^{1} r \tilde{\Psi}_{i}(r) U(r) d r . \frac{d C_{f}(z)}{d z}
$$

The inlet boundary condition given by Eq. (9b) is transformed through the operator $\int_{0}^{1} r U(r) \tilde{\Psi}_{i}(r)() d r$, to provide

$$
\bar{C}_{h, i}(0)=\left(1-C_{f}(0)\right) \int_{0}^{1} r \tilde{\Psi}_{i}(r) U(r) d r
$$

Equations (13a)-(13c) form a coupled nonlinear system of ordinary differential equations that are numerically solved by appropriate computational routines, such as the NDSolve intrinsic function of the Mathematica system (Wolfram, 2015). After the numerical solution procedure is concluded, the concentration profile is recovered through its respective inversion formulae and the proposed nonlinear filter solution.

After the solution of the concentration field is available, through either one of the solution variants, the average solute concentration along the channel can be analytically derived through the following relation:

$$
C_{a v}(z)=\frac{\int_{0}^{1} r U(r) C(r, z) d r}{\int_{0}^{1} r U(r) d r}
$$

\section{RESULTS AND DISCUSSION}

The results presented in this section were obtained through a mixed symbolic-numerical computational routine built on the Mathematica v.10 platform, and employing the subroutine NDSolve for the solution of the nonlinear transformed ODEs systems, Eqs. (5), (6), (13a), and (13c). Numerical results were generated through the two solution variants explored in this work and are compared with results available in the literature, also as a way of numerically verifying the developed computational code.

Tables 1-3 present the convergence behavior of the dimensionless average solute concentration at different positions along the $z$ direction and for increasing truncation orders NT of the transformed ODE system. Two different combinations for the parameter values are investigated to evaluate the influence of the nonlinear term on the convergence of the series solution. Table 1 
TABLE 1: Convergence behavior of the dimensionless average solute concentration $C_{a v}(z)$ for the model proposed by Urtiaga et al. (1992)

\begin{tabular}{|c|c|c|c|c|c|c|c|}
\hline \multicolumn{8}{|c|}{ GITT with nonlinear eigenvalue problem } \\
\hline \multicolumn{4}{|c|}{$\mathrm{Sh}_{w}=0.1$ and $\gamma=10$} & \multicolumn{4}{|c|}{$\mathrm{Sh}_{w}=10$ and $\gamma=1$} \\
\hline NT & $z=0.01$ & $z=0.5$ & 2 & NT & $=0.01$ & $z=0.5$ & $z=\mathbf{2}$ \\
\hline 10 & & 0.590 & 0.218047 & & & 80 & 509 \\
\hline 20 & & & & & & 54 & \\
\hline 30 & & & & & & & \\
\hline 40 & & & & & & & \\
\hline 50 & & & & & & & \\
\hline 60 & & 0.590917 & & 60 & 0.9 & 0.174963 & 508 \\
\hline \multicolumn{8}{|c|}{ GITT with nonlinear filter } \\
\hline \multicolumn{4}{|c|}{$\mathrm{Sh}_{w}=0.1$ and $\gamma=10$} & \multicolumn{4}{|c|}{$\mathrm{Sh}_{w}=10$ and $\gamma=1$} \\
\hline NT & $z=0.01$ & $z=0.5$ & $z=2$ & NT & $z=0.01$ & $z=0.5$ & $z=2$ \\
\hline 10 & 0.983645 & 0.590924 & 0.218046 & 20 & 0.922889 & 0.174971 & 0.001509 \\
\hline 20 & 0.983602 & 0.590918 & 0 & 40 & 0.922822 & 0.174965 & 0.001508 \\
\hline 30 & 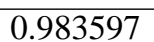 & 0.590917 & 6 & 60 & 0.922811 & 0.174964 & 1508 \\
\hline 40 & & 0.590 & & 80 & 0.922 & 0.174963 & 508 \\
\hline 50 & & 0.590 & & 10 & 0.922805 & 0.174963 & 508 \\
\hline 60 & 0.98 & 0.590917 & 046 & 120 & 0.922804 & 0.174963 & 0.001508 \\
\hline ef.* & 0.9835 & 0.5903 & 0.2177 & - & 0.9227 & 0.1749 & 0.0015 \\
\hline
\end{tabular}

(*) Cardoso et al. (2009).

TABLE 2: Convergence behavior of the dimensionless average solute concentration $C_{a v}(z)$ for the model proposed by Kim and Stroeve (1988)

\begin{tabular}{|c|c|c|c|c|c|c|c|}
\hline \multicolumn{8}{|c|}{ GITT with nonlinear eigenvalue problem } \\
\hline \multicolumn{4}{|c|}{$\mathrm{Sh}_{w}=1, \alpha=15$, and $\beta=1000$} & \multicolumn{4}{|c|}{$\mathrm{Sh}_{w}=10, \alpha=1000$, and $\beta=15$} \\
\hline NT & $z=0.05$ & $z=0.5$ & $z=2$ & NT & $z=0.05$ & $z=0.5$ & $z=2$ \\
\hline 10 & & & & & & 7 & 20 \\
\hline 20 & & & & & & & \\
\hline 30 & & & & & & & \\
\hline 40 & & & & 40 & & & 546 \\
\hline 50 & & & & 50 & & & 0.000546 \\
\hline 60 & & 576 & 0.052523 & 60 & & & 0.000546 \\
\hline \multicolumn{8}{|c|}{ GITT with nonlinear filter } \\
\hline \multicolumn{4}{|c|}{$\mathrm{Sh}_{w}=1, \alpha=15$, and $\beta=1000$} & \multicolumn{4}{|c|}{$\mathrm{Sh}_{w}=10, \alpha=1000$, and $\beta=15$} \\
\hline NT & $z=0.05$ & $z=0.5$ & $z=2$ & NT & $z=0.05$ & $z=0.5$ & $z=2$ \\
\hline 2 & 923093 & 0.492575 & .052523 & 20 & 0.7 & 0.131654 & 0.000546 \\
\hline 4 & $\overline{3}$ & 0.4 & 3 & 40 & & 0.13 & 0.000546 \\
\hline 6 & $\overline{2}$ & 0. & & 60 & & 0.13 & 0.000546 \\
\hline 8 & 2 & & & 80 & & 0.131 & 0.000546 \\
\hline 10 & & & & 100 & & & 0.000546 \\
\hline 12 & 0.923352 & 0.492575 & 0.052523 & 120 & & 0.131645 & 0.000546 \\
\hline Ref.* & 0.924 & 0.494 & 0.0533 & - & 0.716 & 0.131 & 000055 \\
\hline
\end{tabular}

(*) Kim and Stroeve (1988). 
TABLE 3: Convergence behavior of the dimensionless average solute concentration $C_{a v}(z)$ for the model proposed by Kim and Stroeve (1989)

\begin{tabular}{|c|c|c|c|c|c|c|c|}
\hline \multicolumn{8}{|c|}{ GITT with nonlinear eigenvalue problem } \\
\hline \multicolumn{3}{|c|}{$\mathrm{Sh}_{w}=1, \alpha=15$, and $\beta=1000$} & \multicolumn{3}{c|}{$\mathrm{Sh}_{w}=10, \alpha=1000$, and $\beta=15$} \\
\hline $\mathbf{N T}$ & $\boldsymbol{z = \mathbf { 0 . 0 5 }}$ & $\boldsymbol{z = \mathbf { 0 . 5 }}$ & $\boldsymbol{z = 2}$ & $\mathbf{N T}$ & $\boldsymbol{z}=\mathbf{0 . 0 5}$ & $\boldsymbol{z = \mathbf { 0 . 5 }}$ & $\boldsymbol{z = \mathbf { 2 }}$ \\
\hline 10 & 0.935884 & 0.610327 & 0.238780 & 10 & 0.720639 & 0.137532 & 0.001181 \\
\hline 20 & 0.935852 & 0.610312 & 0.238775 & 20 & 0.720455 & 0.137526 & 0.001181 \\
\hline 30 & 0.935849 & 0.610311 & 0.238774 & 30 & 0.720441 & 0.137528 & 0.001181 \\
\hline 40 & 0.935848 & 0.610310 & 0.238774 & 40 & 0.720439 & 0.137529 & 0.001181 \\
\hline 50 & 0.935848 & 0.610310 & 0.238774 & 50 & 0.720438 & 0.137530 & 0.001181 \\
\hline 60 & 0.935848 & 0.610310 & 0.238774 & 60 & 0.720438 & 0.137530 & 0.001181 \\
\hline
\end{tabular}

presents the results obtained from the mathematical model proposed by Urtiaga et al. (1992), providing also a direct comparison with the earlier work of Cardoso et al. (2009), considering a linear eigenvalue problem without any filtering scheme for the nonlinear boundary condition, but with very high truncation orders (NT up to 1000). Tables 2 and 3 present the results obtained from the mathematical models proposed by Kim and Stroeve (1988; 1989), respectively. The present solution with nonlinear eigenvalue problem provides a marked improvement on convergence rates over the previous more simple GITT implementation (Cardoso et al. 2009) and ensures in all cases five or six fully converged significant digits, in the whole axial variable range analyzed, with truncation orders as low as NT $=40$ or NT $=60$, depending on the parameter values. The solution obtained through the GITT with nonlinear filter has also achieved an excellent convergence rate, reaching again five or six converged significant digits with truncation orders of NT $=40$ or NT $=120$ in each set of results of Table 1 , and six converged significant digits with NT $=12$ terms and five digits with NT $=120$ terms, in each of the two sets of results of Table 2. The results are in excellent agreement with previous results from the literature as well. The present set of results suggests that the simulations with parameters that increase the relative importance of the nonlinear boundary condition term, especially for the second solution variant that employs a linear eigenvalue problem, require higher truncation orders in the transformed system to ensure the same convergence level.

Tables 4-6 provide, for each of the three models, respectively, a convergence analysis of the nonlinear eigenfunction expansion variant only, by considering a fixed maximum value of the truncation order $\left(\mathrm{NT}_{\max }=50\right)$ and inspecting the convergence behavior of both the local concentration $C(r, z)$ and of the average concentration $C_{a v}(z)$, for increasing number of terms in the fixed expansion, for $\mathrm{N}=6$ to 30 , in steps of 6 , for the local field, and $\mathrm{N}=4$ to 20 , in steps of 4 , for the average field. This analysis allows one to verify that the convergence of the eigenfunction expansions is indeed outstanding once the nonlinear eigenvalue problem is adopted as the expansion basis. The local concentration field is fully converged to four or five significant digits, in the worst case, with just 30 terms in the expansion, which occurs for the lower values of the $z$ variable and at $r=1$. As for the average concentration, again in the worst situation for $z=0.01$, five or six significant digits are converged for $\mathrm{N}$ as low as 16 .

Tables 7 and 8 present a comparison of the dimensionless average concentration values between the two hybrid solution schemes explored in this work, against available literature data. The GITT results here reported were obtained with a fixed truncation order for the transformed system, with NT $=60$ for the nonlinear eigenvalue problem solution in both Tables 7 and 8 , and for the nonlinear filter solution, NT $=120$ in Table 7 and NT $=250$ in Table 8. One may observe 
TABLE 4: Convergence behavior of GITT solution with nonlinear eigenvalue problem for the dimensionless local $C(r, z)$ and average $C_{a v}(z)$ solute concentrations, for the model proposed by Urtiaga et al. (1992) with $\mathrm{Sh}_{w}=10$ and $\gamma=1$ and with $\mathrm{NT}_{\max }=50$

\begin{tabular}{|c|c|c|c|c|c|c|}
\hline \multicolumn{7}{|c|}{$C(r, z)$} \\
\hline $\mathbf{N}$ & $r=0, z=0.1$ & $r=0 \quad z=0.25$ & $r=0 \quad z=0.5$ & $r=1 z=0.1$ & $r=1 z=0.25$ & $r=1 z=0.5$ \\
\hline 6 & 0.952403 & 0.639705 & 0.289923 & 0.101507 & 0.058996 & 0.027143 \\
\hline 12 & 0.952309 & 0.639657 & 0.289903 & 0.101174 & 0.058827 & 0.027069 \\
\hline 18 & 0.952304 & 0.639655 & 0.289902 & 0.101139 & 0.058809 & 0.027061 \\
\hline 24 & 0.952303 & 0.639654 & 0.289901 & 0.101130 & 0.058805 & 0.027059 \\
\hline 30 & 0.952302 & 0.639654 & 0.289901 & 0.101127 & 0.058803 & 0.027059 \\
\hline \multicolumn{7}{|c|}{$C_{a v}(z)$} \\
\hline $\mathbf{N}$ & $z=0.01$ & $z=0.1$ & $z=0.2$ & $z=0.5$ & $z=1$ & $z=2$ \\
\hline 4 & 0.922013 & 0.636334 & 0.455869 & 0.174953 & 0.035824 & 0.001508 \\
\hline 8 & 0.922798 & 0.636373 & 0.455894 & 0.174963 & 0.035826 & 0.001508 \\
\hline 12 & 0.922802 & 0.636374 & 0.455895 & 0.174963 & 0.035826 & 0.001508 \\
\hline 16 & 0.922803 & 0.636374 & 0.455895 & 0.174963 & 0.035826 & 0.001508 \\
\hline 20 & 0.922803 & 0.636374 & 0.455895 & 0.174963 & 0.035826 & 0.001508 \\
\hline Ref.** & 0.9227 & 0.6363 & 0.4558 & 0.1749 & 0.0358 & 0.00150 \\
\hline
\end{tabular}

(*) Cardoso et al. (2009).

TABLE 5: Convergence behavior of GITT solution with nonlinear eigenvalue problem for the dimensionless local $C(r, z)$ and average $C_{a v}(z)$ solute concentrations, for the model proposed by Kim and Stroeve (1988) with $\mathrm{Sh}_{w}=1, \alpha=15$, and $\beta=1000$ and with $\mathrm{NT}_{\max }=50$

\begin{tabular}{|c|c|c|c|c|c|c|}
\hline \multicolumn{7}{|c|}{$\boldsymbol{C}(\boldsymbol{r}, \boldsymbol{z})$} \\
\hline $\mathbf{N}$ & $\boldsymbol{r}=\mathbf{0} \boldsymbol{z}=\mathbf{0 . 1}$ & $\boldsymbol{r}=\mathbf{0} \boldsymbol{z = \mathbf { 0 . 2 5 }}$ & $\boldsymbol{r = \mathbf { 0 } \boldsymbol { z } = \mathbf { 0 . 5 }}$ & $\boldsymbol{r}=\mathbf{1} \boldsymbol{z = \mathbf { 0 . 1 }}$ & $\boldsymbol{r = 1} \boldsymbol{z = \mathbf { 0 . 2 5 }}$ & $\boldsymbol{r = 1} \boldsymbol{z = \mathbf { 0 . 5 }}$ \\
\hline 6 & 0.986177 & 0.848812 & 0.606689 & 0.598623 & 0.466586 & 0.326950 \\
\hline 12 & 0.986136 & 0.848790 & 0.606675 & 0.598471 & 0.466505 & 0.326896 \\
\hline 18 & 0.986134 & 0.848789 & 0.606674 & 0.598457 & 0.466497 & 0.326891 \\
\hline 24 & 0.986133 & 0.848789 & 0.606674 & 0.598454 & 0.466496 & 0.326890 \\
\hline 30 & 0.986133 & 0.848789 & 0.606674 & 0.598453 & 0.466495 & 0.326890 \\
\hline \multicolumn{7}{|c|}{$\boldsymbol{C}_{\boldsymbol{a} \boldsymbol{v}} \boldsymbol{( z )}$} \\
\hline $\mathbf{N}$ & $\boldsymbol{z}=\mathbf{0 . 0 1}$ & $\boldsymbol{z} \mathbf{\mathbf { 0 . 1 }}$ & $\boldsymbol{z = \mathbf { 0 . 2 }}$ & $\boldsymbol{z}=\mathbf{0 . 5}$ & $\boldsymbol{z}=\mathbf{1}$ & $\boldsymbol{z}=\mathbf{2}$ \\
\hline 4 & 0.982297 & 0.858469 & 0.746044 & 0.492565 & 0.243867 & 0.052522 \\
\hline 8 & 0.982703 & 0.858496 & 0.746061 & 0.492575 & 0.243872 & 0.052523 \\
\hline 12 & 0.982705 & 0.858497 & 0.746062 & 0.492575 & 0.243873 & 0.052523 \\
\hline 16 & 0.982706 & 0.858497 & 0.746062 & 0.492575 & 0.243873 & 0.052523 \\
\hline 20 & 0.982706 & 0.858497 & 0.746062 & 0.492575 & 0.243873 & 0.052523 \\
\hline Ref.* & 0.983 & 0.859 & 0.747 & 0.494 & 0.245 & 0.0533 \\
\hline
\end{tabular}

(*) Kim and Stroeve (1988).

the expected excellent agreement between the two converged GITT solutions, which provide a verification of the purely numerical results of Urtiaga et al. (1992) and Kim and Stroeve (1988), with an adherence to at least two significant digits in all positions and parameter values considered.

Volume 30, Issues 2-3, 2018 
TABLE 6: Convergence behavior of GITT solution with nonlinear eigenvalue problem for the dimensionless local $C(r, z)$ and average $C_{a v}(z)$ solute concentrations, for the model proposed by Kim and Stroeve (1989) with $\mathrm{Sh}_{w}=1, \alpha=15$ and $\beta=1000$ and with $\mathrm{NT}_{\max }=50$

\begin{tabular}{|c|c|c|c|c|c|c|}
\hline \multicolumn{7}{|c|}{$C(r, z)$} \\
\hline $\mathbf{N}$ & $r=0 z=0.1$ & $r=0 z=0.25$ & $r=0 \quad z=0.5$ & $r=1 z=0.1$ & $r=1 z=0.25$ & $r=1 z=0.5$ \\
\hline 6 & 0.988226 & 0.878357 & 0.694949 & 0.679516 & 0.587795 & 0.487726 \\
\hline 12 & 0.988199 & 0.878344 & 0.694940 & 0.679417 & 0.587744 & 0.487690 \\
\hline 18 & 0.988197 & 0.878343 & 0.694939 & 0.679408 & 0.587740 & 0.487687 \\
\hline 24 & 0.988197 & 0.878343 & 0.694939 & 0.679406 & 0.587738 & 0.487687 \\
\hline 30 & 0.988197 & 0.878343 & 0.694939 & 0.679405 & 0.587738 & 0.487686 \\
\hline \multicolumn{7}{|c|}{$C_{a v}(z)$} \\
\hline $\mathbf{N}$ & $z=0.01$ & $z=0.1$ & $z=0.2$ & $z=0.5$ & $z=1$ & $z=2$ \\
\hline 4 & 0.984321 & 0.884744 & 0.798782 & 0.610303 & 0.421349 & 0.238772 \\
\hline 8 & 0.984678 & 0.884761 & 0.798793 & 0.610310 & 0.421353 & 0.238774 \\
\hline 12 & 0.984680 & 0.884762 & 0.798793 & 0.610310 & 0.421353 & 0.238774 \\
\hline 16 & 0.984680 & 0.884762 & 0.798793 & 0.610310 & 0.421353 & 0.238774 \\
\hline 20 & 0.984680 & 0.884762 & 0.798793 & 0.610310 & 0.421353 & 0.238774 \\
\hline
\end{tabular}

This comparative analysis is complemented through Figs. 2-4, which provide the profiles of the dimensionless average solute concentration along the length of the hollow fiber membrane, for the three different models, respectively, and for different values of the parameters. The GITT results were obtained with a truncation order for the transformed system of NT $=50$. To the graphical scale, it is quite clear that the two hybrid solution schemes here employed are coincident, and in excellent agreement with the previously reported results (Urtiaga et al. 1992; Kim and Stroeve, 1988; 1989). It is also clear that the average concentration of the solute is strongly influenced by the values of $\mathrm{Sh}_{w}, \gamma, \alpha$, and $\beta$. The nonlinear filtering solution here adopted leads to a complex domain solution for values of $\gamma$ less than zero. For this reason, in the following graphs, there are no curves of the nonlinear filter solution for negative values of $\gamma$. The solution scheme with the nonlinear eigenvalue problem, here emphasized, does not have this sort of limitation, being valid for any value of $\gamma$. The nonlinear filter solution was not implemented for the third model (Kim and Stroeve 1989), and for this reason only the nonlinear eigenvalue problem solution is provided in Figs. 4(a) and 4(b).

Figures 2(a) and 2(b) illustrate the influence of the parameter $\gamma$ on the average concentration of solute for the Sherwood numbers $\mathrm{Sh}_{w}=0.1$ and $\mathrm{Sh}_{w}=1$, respectively. $\gamma$ is related to the variable distribution coefficient depending on the solute concentration along the separator. It is possible to observe that with the increase of $\gamma$, there is a considerable improvement in the mass transfer rates of the solute through the membrane. For positive value of $\gamma$, the variable distribution coefficient is higher at the entrance of the separator where the solute concentration is maximal and it decreases along the channel as the solute concentration decreases. For negative value of $\gamma$ an opposite effect occurs.

Figures 3 and 4 illustrate the effect of the maximum facilitation factor $\alpha$ and the dimensionless equilibrium constant $\beta$ on the average concentration of the solute in the membrane. $\alpha$ is defined as the ratio of carrier-facilitated transport to purely physical transport of solute. High values of $\alpha$ ensure a more efficient transport of the solute through the membrane, according to Figs. 3(a) and 4(a). $\beta$ gives a measure of the intensity of the complexation reaction and has an 
TABLE 7: Comparison of the dimensionless average solute concentration $C_{a v}(z)$ with data from Urtiaga et al. (1992)

\begin{tabular}{|c|c|c|c|c|c|c|}
\hline & \multicolumn{3}{|c|}{$\mathbf{S h}_{w}=0.1$ and $\gamma=0$} & \multicolumn{3}{|c|}{$\mathrm{Sh}_{w}=0.1$ and $\gamma=0.1$} \\
\hline$Z$ & $\begin{array}{l}\text { Urtiaga et } \\
\text { al. (1992) }\end{array}$ & $\begin{array}{l}\text { Nonlinear } \\
\text { filter }\end{array}$ & $\begin{array}{c}\text { Nonlinear } \\
\text { eigenvalue } \\
\text { problem }\end{array}$ & $\begin{array}{l}\text { Urtiaga et } \\
\text { al. (1992) }\end{array}$ & $\begin{array}{l}\text { Nonlinear } \\
\text { filter }\end{array}$ & $\begin{array}{c}\text { Nonlinear } \\
\text { eigenvalue } \\
\text { problem }\end{array}$ \\
\hline 0.01 & 0.997937 & 0.998034 & 0.998034 & 0.997739 & 0.997844 & 0.997844 \\
\hline 0.1 & 0.980597 & 0.980814 & 0.980814 & 0.978829 & 0.979062 & 0.979062 \\
\hline 0.2 & 0.961899 & 0.962184 & 0.962185 & 0.958534 & 0.958842 & 0.958842 \\
\hline 0.5 & 0.908104 & 0.908536 & 0.908536 & 0.900555 & 0.901012 & 0.901012 \\
\hline 1.0 & 0.825084 & 0.825714 & 0.825714 & 0.812170 & 0.812824 & 0.812824 \\
\hline 2.0 & 0.682032 & 0.682031 & 0.682032 & 0.660485 & 0.662879 & 0.662879 \\
\hline & \multicolumn{3}{|c|}{$S h_{w}=0.1$ and $\gamma=1$} & \multicolumn{3}{|c|}{$\mathbf{S h}_{w}=1$ and $\gamma=0$} \\
\hline$Z$ & $\begin{array}{l}\text { Urtiaga et } \\
\text { al. (1992) }\end{array}$ & $\begin{array}{l}\text { Nonlinear } \\
\text { filter }\end{array}$ & $\begin{array}{c}\text { Nonlinear } \\
\text { eigenvalue } \\
\text { problem }\end{array}$ & $\begin{array}{l}\text { Urtiaga et } \\
\text { al. (1992) }\end{array}$ & $\begin{array}{l}\text { Nonlinear } \\
\text { filter }\end{array}$ & $\begin{array}{c}\text { Nonlinear } \\
\text { eigenvalue } \\
\text { problem }\end{array}$ \\
\hline 0.01 & 0.995759 & 0.996195 & 0.996195 & 0.982283 & 0.982961 & 0.982961 \\
\hline 0.1 & 0.963544 & 0.964428 & 0.964428 & 0.859620 & 0.860584 & 0.860585 \\
\hline 0.2 & 0.933780 & 0.931542 & 0.931542 & 0.748813 & 0.749808 & 0.749808 \\
\hline 0.5 & 0.840861 & 0.842418 & 0.842418 & 0.499920 & 0.500057 & 0.500057 \\
\hline 1.0 & 0.716369 & 0.718403 & 0.718403 & 0.254408 & 0.255004 & 0.255004 \\
\hline \multirow[t]{2}{*}{2.0} & 0.532879 & 0.535389 & 0.535389 & 0.066080 & 0.066316 & 0.066316 \\
\hline & \multicolumn{3}{|c|}{$\mathbf{S h}_{w}=1$ and $\gamma=0.1$} & \multicolumn{3}{|c|}{$\mathbf{S h}_{w}=1$ and $\gamma=1$} \\
\hline$Z$ & $\begin{array}{l}\text { Urtiaga et } \\
\text { al. (1992) }\end{array}$ & $\begin{array}{l}\text { Nonlinear } \\
\text { filter }\end{array}$ & $\begin{array}{c}\text { Nonlinear } \\
\text { eigenvalue } \\
\text { problem }\end{array}$ & $\begin{array}{l}\text { Urtiaga et } \\
\text { al. (1992) }\end{array}$ & $\begin{array}{l}\text { Nonlinear } \\
\text { filter }\end{array}$ & $\begin{array}{c}\text { Nonlinear } \\
\text { eigenvalue } \\
\text { problem }\end{array}$ \\
\hline 0.01 & 0.980048 & 0.981756 & 0.981756 & 0.971118 & 0.973046 & 0.973046 \\
\hline 0.1 & 0.851779 & 0.854148 & 0.854148 & 0.811091 & 0.813065 & 0.813065 \\
\hline 0.2 & 0.738177 & 0.740630 & 0.740630 & 0.682304 & 0.684142 & 0.684142 \\
\hline 0.5 & 0.486580 & 0.488829 & 0.488829 & 0.421381 & 0.422825 & 0.422824 \\
\hline 1.0 & 0.245085 & 0.246757 & 0.246757 & 0.198930 & 0.199923 & 0.199923 \\
\hline 2.0 & 0.063000 & 0.063670 & 0.063670 & 0.048622 & 0.049018 & 0.049018 \\
\hline
\end{tabular}

TABLE 8: Comparison of the dimensionless average solute concentration $C_{a v}(z)$ with data from Kim and Stroeve (1988)

\begin{tabular}{|c|c|c|c|c|c|c|}
\hline & \multicolumn{2}{|c|}{$\mathbf{S h}_{\boldsymbol{w}}=\mathbf{0 . 1} \boldsymbol{\alpha}=\mathbf{1 0 0 0}, \mathbf{a n d} \boldsymbol{\beta}=\mathbf{1 5}$} & \multicolumn{3}{c|}{$\mathbf{S h}_{\boldsymbol{w}}=\mathbf{1}, \boldsymbol{\alpha}=\mathbf{1 0 0 0}$, and $\boldsymbol{\beta}=\mathbf{1 5}$} \\
\hline $\boldsymbol{Z}$ & $\begin{array}{c}\text { Kim and } \\
\text { Stroeve } \\
(1988)\end{array}$ & $\begin{array}{c}\text { Nonlinear } \\
\text { filter }\end{array}$ & $\begin{array}{c}\text { Nonlinear } \\
\text { eigenvalue } \\
\text { problem }\end{array}$ & $\begin{array}{c}\text { Kim and } \\
\text { Stroeve } \\
(1988)\end{array}$ & $\begin{array}{c}\text { Nonlinear } \\
\text { filter }\end{array}$ & $\begin{array}{c}\text { Nonlinear } \\
\text { eigenvalue } \\
\text { problem }\end{array}$ \\
\hline $\mathbf{0 . 0 5}$ & 0.730 & 0.730308 & 0.730308 & 0.717 & 0.716858 & 0.716859 \\
\hline $\mathbf{0 . 1}$ & 0.592 & 0.591781 & 0.591780 & 0.580 & 0.579569 & 0.579569 \\
\hline $\mathbf{0 . 2}$ & 0.407 & 0.406383 & 0.406383 & 0.396 & 0.396051 & 0.396052 \\
\hline $\mathbf{0 . 5}$ & 0.138 & 0.137588 & 0.137588 & 0.132 & 0.132066 & 0.132066 \\
\hline $\mathbf{1 . 0}$ & 0.023 & 0.022720 & 0.022720 & 0.021 & 0.021277 & 0.021277 \\
\hline $\mathbf{2 . 0}$ & 0.00062 & 0.000619 & 0.000619 & 0.00055 & 0.000552 & 0.000552 \\
\hline
\end{tabular}

Volume 30, Issues 2-3, 2018 


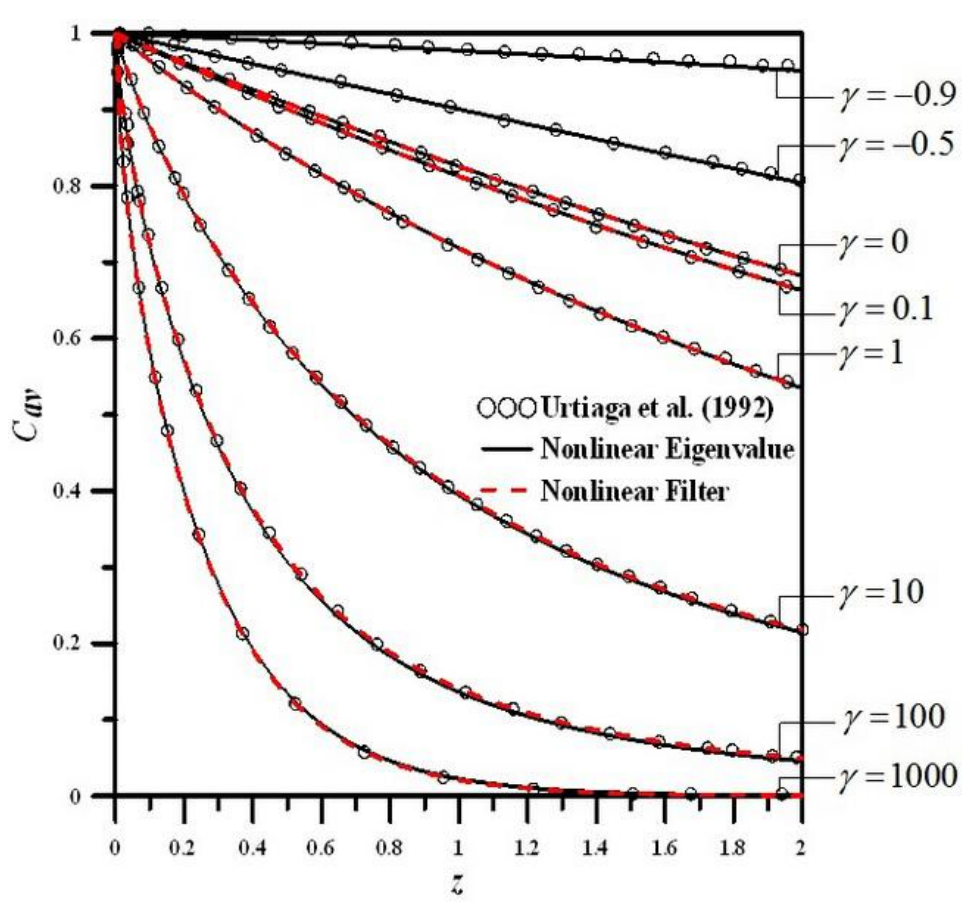

(a)

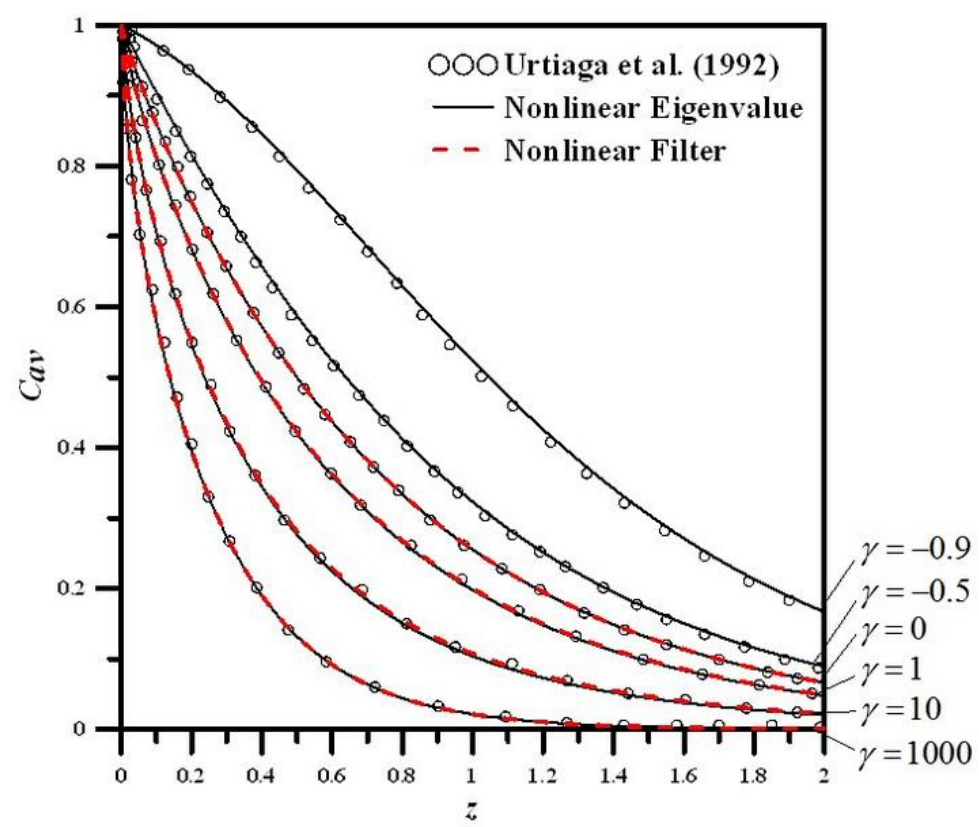

(b)

FIG. 2: (a) Effect of parameter $\gamma$ on dimensionless average solute concentration for the model proposed by Urtiaga et al. (1992) with $\mathrm{Sh}_{w}=0.1$; (b) Effect of parameter $\gamma$ on dimensionless average solute concentration for the model proposed by Urtiaga et al. (1992) with $\mathrm{Sh}_{w}=1$ 


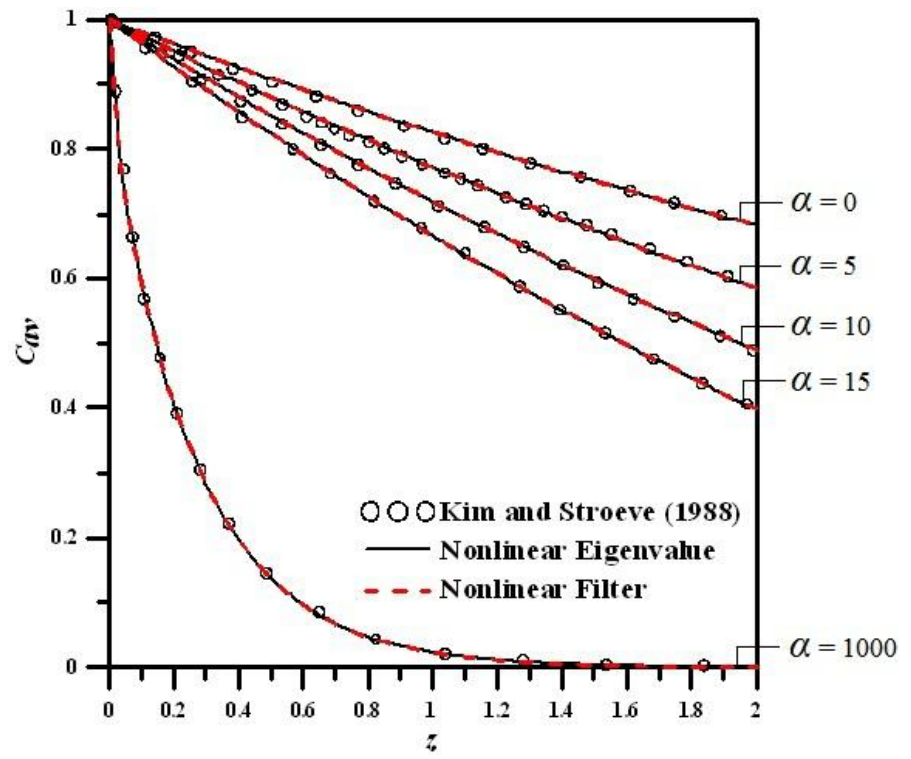

(a)

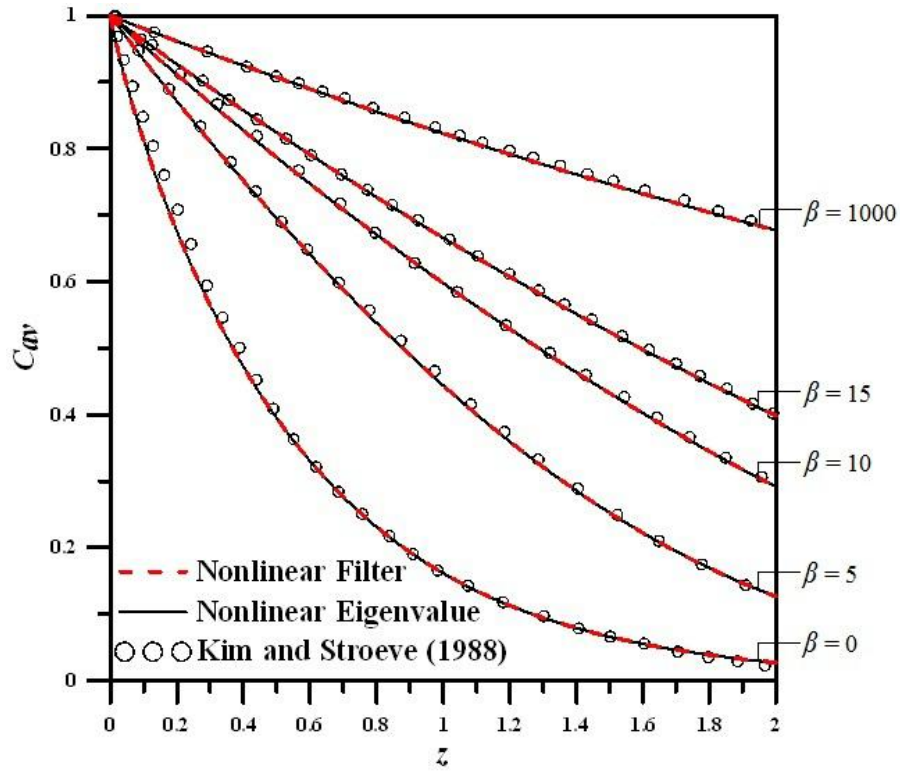

(b)

FIG. 3: (a) Effect of parameter $\alpha$ on dimensionless average solute concentration for the model proposed by Kim and Stroeve (1988) with $\mathrm{Sh}_{w}=0.1$ and $\beta=15$; (b) Effect of parameter $\beta$ on dimensionless average solute concentration for the model proposed by Kim and Stroeve (1988) with $\mathrm{Sh}_{w}=0.1$ and $\alpha=15$

opposite effect to that of $\alpha$. High values of $\beta$ indicate a saturation in the complexation reaction and make it difficult to transport the solute, according to Figs. 3(b) and 4(b).

Volume 30, Issues 2-3, 2018 


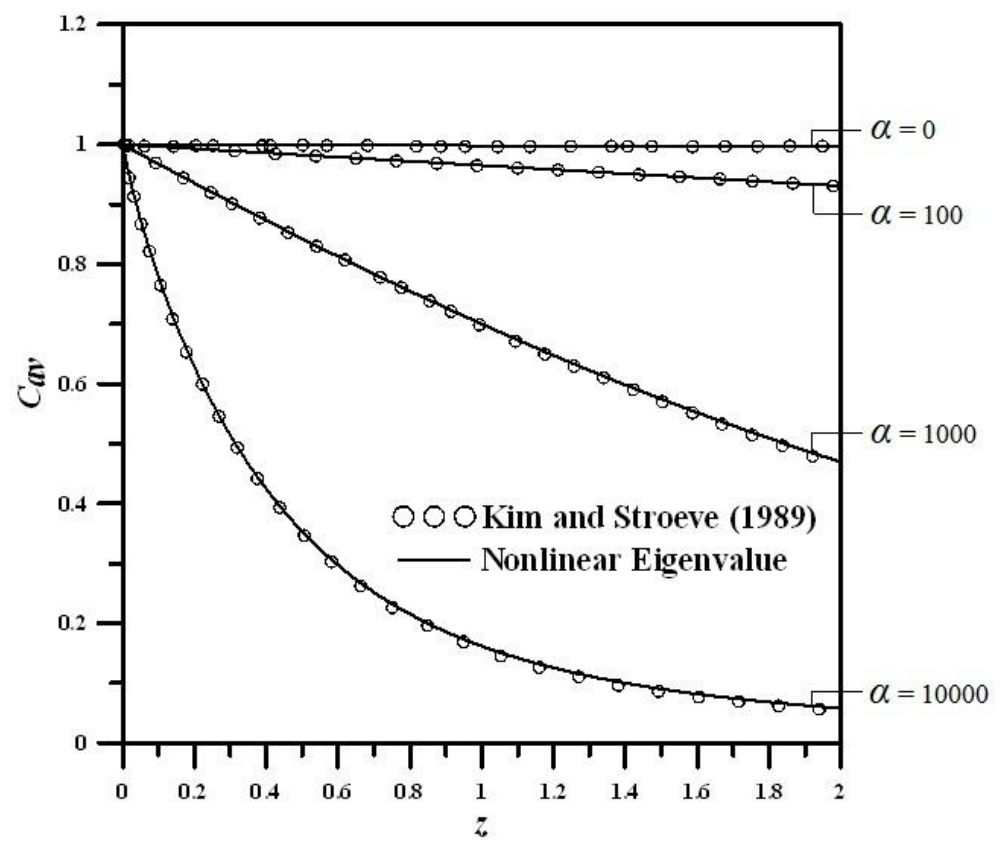

(a)

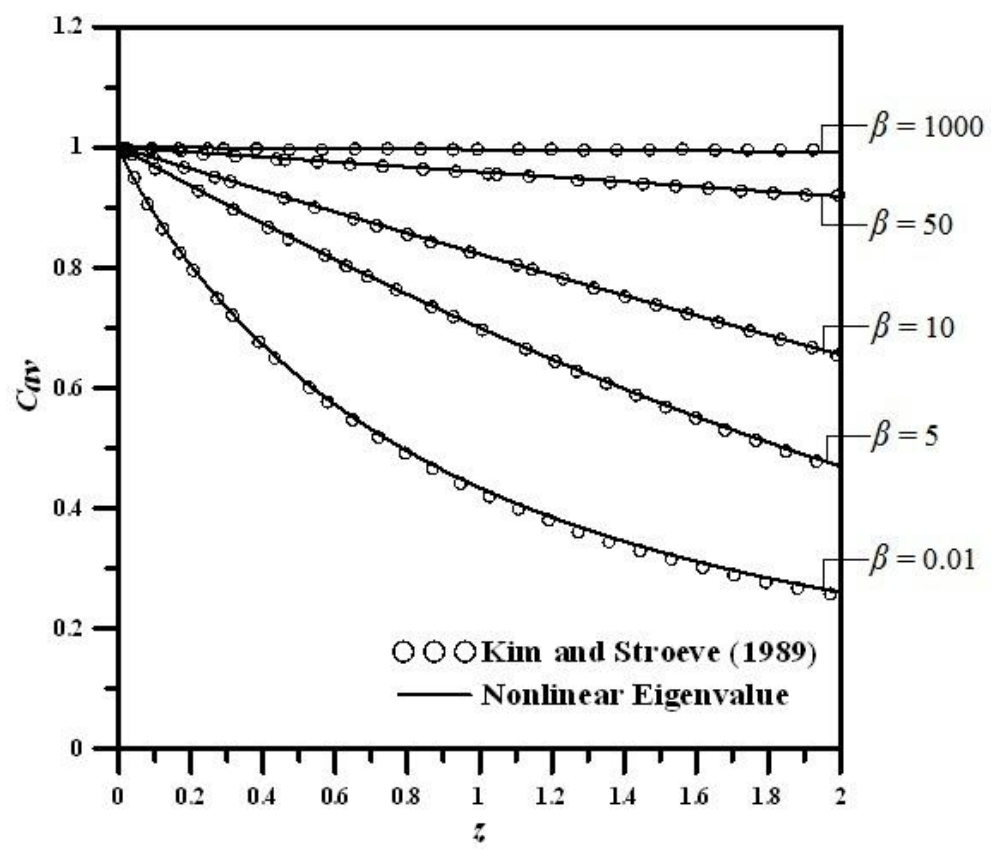

(b)

FIG. 4: (a) Effect of parameter $\alpha$ on dimensionless average solute concentration for the model proposed by Kim and Stroeve (1989) with $\mathrm{Sh}_{w}=0.001$ and $\beta=5$; (b) Effect of parameter $\beta$ on dimensionless average solute concentration for the model proposed by Kim and Stroeve (1989) with $\mathrm{Sh}_{w}=0.001$ and $\alpha=1000$ 


\section{CONCLUSIONS}

A nonlinear convection-diffusion mathematical model for the mass transport of a solute through hollow fiber membranes is here analyzed. The Generalized Integral Transform Technique (GITT) is applied to solve the mathematical model considering two proposals for the eigenvalue problem: a formulation that does not account for curvature and velocity profile coefficients but incorporates the nonlinearity of the boundary condition, and must then be simultaneously solved with the set of ordinary differential equations for the transformed concentration field, and another formulation with a linear eigenvalue problem that includes geometry and velocity field information, but does not account for the nonlinear boundary condition. This second alternative then requires the development of a nonlinear filtering solution to homogenize the original nonlinear boundary condition, moving the nonlinear effect to a new nonlinear source term in the convection-diffusion equation itself. Both hybrid solution schemes, either with the nonlinear eigenvalue problem or with the nonlinear filter, have markedly improved convergence rates with respect to the plain GITT solution with a linear eigenvalue problem and without any filter, previously implemented with very high truncation orders. The proposed solution with a nonlinear eigenvalue problem reaches six significant digits convergence with truncation orders as low as 40 to 60 terms. The agreement between the two GITT solutions schemes is remarkable, for different values of the governing parameters, as well as with other available purely numerical solutions of the same problem. The implemented nonlinear filter solution does have some limitation in certain range of the governing parameters, and requires that other possible filters be searched for. The proposed GITT approach with nonlinear eigenvalue problem results from a general methodology for convection-diffusion problems with nonlinear boundary conditions. It may even be further improved in terms of convergence rate, by either considering an eigenvalue problem that incorporates all the original spatial operators of the partial differential equation and/or by considering complementary convergence enhancement techniques, such as an analytical filtering solution to reduce the importance of source terms eventually present in the problem formulation.

\section{ACKNOWLEDGMENTS}

The authors acknowledge the financial support provided by FAPERJ, CNPq, and CAPES, all of them research sponsoring agencies in Brazil. R.M.C. acknowledges the financial support provided by the Leverhulme Trust Fund for a Visiting Professorship at the Mechanical Eng. Dept., University College London (U.C.L.), U.K. C.P.N.C. acknowledges the Visiting Researcher fellowship for a one year stay at U.C.L., U.K., provided by CAPES/INMETRO, Brazil.

\section{REFERENCES}

Cardoso, S.A., Macedo, E.N., Quaresma, J.N.N., and Cotta, R.M., Hybrid Solutions for the Analysis of Mass Transfer in Hollow-Fiber Membranes, in Proc. COBEM 2009 20th Intl. Cong. Mech. Eng., Gramado, RS, Brazil, November 15-20, 2009.

Cotta, R.M., Integral Transforms in Computational Heat and Fluid Flow, Boca Raton, FL: CRC Press, 1993.

Cotta, R.M., Benchmark Results in Computational Heat and Fluid Flow: The Integral Transform Method, Int. J. Heat Mass Transf., vol. 37, pp. 381-393, 1994.

Cotta, R.M., The Integral Transform Method in Thermal and Fluids Sciences and Engineering, New York: Begell House, 1998.

Volume 30, Issues 2-3, 2018 
Cotta, R.M. and Mikhailov, M.D., Heat Conduction: Lumped Analysis, Integral Transforms, Symbolic Computation, Chichester, UK: Wiley-Interscience, 1997.

Cotta, R.M. and Mikhailov, M.D., Hybrid Methods and Symbolic Computations, in Handbook of Numerical Heat Transfer, 2nd ed., W.J. Minkowycz, E.M. Sparrow, and J.Y. Murthy, Eds., New York: John Wiley, 2006.

Cotta, R.M., Hybrid Numerical-Analytical Approach to Nonlinear Diffusion Problems, Numer. Heat Transf. Part B., vol. 127, pp. 217-226, 1990.

Cotta, R.M., Knupp, D.C., and Naveira-Cotta, C.P., Analytical Heat and Fluid Flow in Microchannels and Microsystems: Mechanical Engineering Series, New York: Springer, 2016a.

Cotta, R.M., Naveira-Cotta, C.P., and Knupp, D.C., Nonlinear Eigenfunction Expansions for the Solution of Nonlinear Diffusion Problems, in Proc. of 1st Therm. Fluid Eng. Summer Conf., TFESC, August 9-12, New York City, NY, 2015.

Cotta, R.M., Naveira-Cotta, C.P., and Knupp, D.C., Nonlinear Eigenvalue Problem in the Integral Transforms Solution of Convection-Diffusion with Nonlinear Boundary Condition, Int. J. Numer. Methods Heat Fluid Flow, vol. 26, nos. 3-4, pp. 767-789, 2016b.

Hosseini, S.S., Bringas, E., Tan, N.R., Ortiz, I., Ghahramani, M., and Shahmirzadi, M.A.A., Recent Progress in Development of High Performance Polymeric Membranes and Materials for Metal Plating Wastewater Treatment: A Review, J. Water Process Eng., vol. 9, pp. 78-110, 2016.

Kakaç, S., Yener, Y., and Naveira-Cotta, C.P., Heat Conduction, 5th ed., Oxford, U.K.: Taylor and Francis, 2018.

Kim, J.-I. and Stroeve, P., Mass Transfer in Separation Devices with Reactive Hollow Fibers, Chem. Eng. Sci., vol. 41, pp. 247-257, 1988.

Kim, J.-I. and Stroeve, P., Selective and Enhanced Mass Separation in Hollow Fiber Membranes with Facilitated Ion-Pair Transport, J. Membr. Sci., vol. 45, pp. 99-114, 1989.

Kim, J.-I. and Stroeve, P., Uphill Transport of a Dilute Solute in Mass Separation Devices with CoTransport Membranes, J. Membr. Sci., vol. 49, pp. 37-53, 1990.

Kocherginsky, N.M., Yang, Q., and Seelam, L., Recent Advances in Supported Liquid Membrane Technology, Sep. Purif. Technol., vol. 53, pp. 171-177, 2007.

Lakshmi, D.S., Muthukumar, M., and Mohan, D., Extraction of Zinc(II) using Liquid Membrane and Performance Optimization using Response Surface Methodology, J. Sci. Ind. Res., vol. 63, no. 1, pp. 74-79, 2004.

Mikhailov, M.D. and Ozisik, M.N., Unified Analysis and Solution in Heat and Mass Diffusion, New York: Wiley, 1984; Dover Publications, 1994.

Noble, R.D., Shape Factors in Facilitated Transport through Membranes, Ind. Eng. Chem. Fund., vol. 22, pp. 139-144, 1983.

Özisik, M.N., Heat Conduction, 2nd ed., New York: John Wiley \& Sons, 1993.

Pontes, P.C., Almeida, A.P., Naveira-Cotta, C.P., and Cotta, R.M., Nonlinear Eigenvalue Problem Approach in the Integral Transforms Analysis of Metal Separation by Polymeric Diffusive Membranes, Proc. CHT17, ICHMT Int. Sympos. Adv. Comput. Heat Transf., Napoli, Italy, May 28-June 1, 2017.

Porter, M.C., Handbook of Industrial Membrane Technology, New York: Noyes Publications, 1990.

Rdzaneck, P., Marszalek, J., and Kaminski, W., Biobutanol Concentration by Pervaporation using Supported Ionic Liquid Membranes, Sep. Purif. Technol., vol. 196, pp. 124-131, 2018.

Swain, B., Sarangi, K., and Das, R.P., Separation of Cadmium and Zinc by Supported Liquid Membrane using TOPS-99 as Mobile Carrier, Sep. Sci. Technol., vol. 39, no. 9, pp. 2171-2188, 2004.

Urtiaga, A.M., Irabien, J.A., and Stroeve, P., Effect of a Variable Solute Distribution Coefficient on Mass Separation in Hollow Fibers, Ind. Eng. Chem. Res., vol. 31, pp. 1362-1366, 1992.

Wolfram, S., The Mathematica Book, Champaign, IL: Wolfram Media, 2015. 